\title{
A Method for Solving Fuzzy Transportation Problem (FTP) using Fuzzy Russell's Method
}

\author{
S. Narayanamoorthy \\ Department of Applied Mathematics, Bharathiar University,Coimbatore - 46, India \\ Email: snm_phd@yahoo.co.in \\ S.Saranya \& S.Maheswari \\ Department of Applied Mathematics, Bharathiar University, Coimbatore - 46, India \\ Email:mathsaran66@gmail.com,smaheswari28@gmail.com
}

\begin{abstract}
The basic transportation problem was originally developed by Hitchcock. In the literature several methods are proposed for solving Fuzzy transportation problem. In this paper, we propose a new algorithm called Fuzzy Russell's method for the initial basic feasible solution to a Fuzzy transportation problem. To examine the proposed method a numerical example is solved. Fuzzy numbers may be normal or abnormal, triangular or trapezoidal or any LR fuzzy number. We can use this proposed method for any kind of Fuzzy numbers.
\end{abstract}

Index Terms - Fuzzy Transportation Problem, Trapezoidal Number, Fuzzy Russell's Method

\section{Introduction}

In Mathematics and Economics, transportation theory is a name given to the study of optimal transportation and allocation of resources. The problem was formalized by the French Mathematician Gaspard Monge in 1781. Tolstoi was one of the first to study the transportation problem mathematically. The transportation problem (TP) refers to a special class of linear programming problem. When the theory of fuzzy sets was first introduced by Zadeh[18].

The transportation problem can be modeled as a standard linear programming problem, which can then be solved by the simplex method. In a typical problem a product is to be transported from $m$ sources to $n$ designations and their capacities are $a_{1}, a_{2} \ldots a_{m}$ and $b_{1}$, $b_{2} \ldots b_{n}$ respectively.

In addition there is a penalty $\mathrm{c}_{\mathrm{ij}}$ associated with transporting unit of product from source i to destination j. This penalty may be cost or delivery time or safety of delivery etc.

A variable $\mathrm{x}_{\mathrm{ij}}$ represents the unknown quantity to be shipped from source $\mathrm{i}$ to destination $\mathrm{j}$. Let $a_{i}$ be the amount of the product available at origin $\mathrm{i}$ and $b_{j}$ be the amount of the product required at destination $\mathrm{j}$.

If shipping cost, are assumed to be proportional to the amount shipped from each origin to each destination so as to minimize total shipping cost turns out to be a linear programming problem. A fuzzy transportation problem is a transportation problem in which the transportation costs, supply and demand quantities are fuzzy quantities. Most of the existing techniques provide only crisp solutions for the fuzzy transportation problem. Shiang-Tai Liu and Chiang Kao [14],Chanas et al. [3], Chanas and Kuchta [2], proposed a method for solving fuzzy transportation problem. Nagoor Gani and Abdul Rezak [11] obtained a fuzzy solution for a two stage cost minimizing fuzzy transportation problem in which supplies and demands are trapezoidal fuzzy numbers. Pandian et al. [13], proposed a method namely, zero point method, for finding a fuzzy optimal solution for a fuzzy transportation problem where all parameters are trapezoidal fuzzy numbers. Amarpreet kaur[1] proposed a new method for solving fuzzy transportation problem by assuming that a decision maker is uncertain about the precise values of the transportation cost, availability and demand of the product. In many fuzzy decision problems, the data are represented in terms of fuzzy numbers.

In a fuzzy transportation problem, all parameters are fuzzy numbers. Fuzzy numbers may be normal or abnormal, triangular or trapezoidal. Ranking method is used to change the fuzzy number into crisp form. The method for ranking was first proposed by Jain [8]. Yager [17] proposed four indices which may be employed for the purpose of ordering fuzzy quantities in $[0,1]$. Further references in this direction can be found in [4-5, 9-10]. Ranking function is used in different areas of fuzzy optimization.

In real life, there are many diverse situations due to uncertainty in judgments, lack of evidence etc. Sometimes it is not possible to get relevant precise data for the cost parameter. This type of imprecise data is 
not always well represented by random variable selected from a probability distribution. Fuzzy number may represent this data.

The aim of fuzzy transportation is to find the least transportation cost of some commodities through a capacitated network when the supply and demand of nodes and the capacity and cost of edges are represented as fuzzy numbers.

There are many methods to find the basic feasible solution, such as North-West Corner Rule, Row Minima Method Column Minima Method, Matrix Minima Method (Lowest Cost Entry Method), Vogel's Approximation Method (Unit Cost Penalty Method) (VAM), Russell's method. Here we use a new method named as Fuzzy Russell's method for solving Fuzzy transportation problem.

Fuzzy Russell's method (Tze-San lee [16]) is probably the best one of the following reasons:

(i) It generates a near-optimal initial feasible solution,

(ii) It simplifies the overall computer code to program it.

This research paper has five sections. In section one is introduction and the development of Fuzzy Transportation problem.

In section two, we just recall the basic concepts and yager's ranking method. In the third section we proposed a new computational procedure for fuzzy Russell's method. In section four, a numerical example is provided to illustrate the algorithm developed in this research paper. Finally we give conclusion in section five.

\section{Preliminaris}

In this section the basic concepts of Fuzzy number, Trapezoidal fuzzy number, Properties of Trapezoidal number, Fuzzy Sets, Crisp Set are recalled.

\subsection{Fuzzy Number}

A real fuzzy number $\tilde{a}$ is a fuzzy subset of the real number R with membership function $\mu_{\tilde{a}}$ satisfying the following conditions,

$\mu_{\tilde{a}}$ is continuous from $\mathrm{R}$ to the closed interval $[0,1]$

$>\mu_{\tilde{a}}$ is strictly increasing and continuous on $\left[\mathrm{a}_{1}, \mathrm{a}_{2}\right]$

$>\mu_{\tilde{a}}$ is strictly decreasing and continuous on $\left[\mathrm{a}_{3}, \mathrm{a}_{4}\right]$

\subsection{Trapezoidal Fuzzy Number}

A fuzzy number $\tilde{A}=(a, b, c, d)$ is said to be a trapezoidal fuzzy number if its membership function is given by,

$$
\mu_{\tilde{A}}=\left\{\begin{array}{ll}
0 & x<m \\
\frac{x-a}{b-a} & a \leq x \leq b \\
\frac{d-x}{d-c} & c \leq x \leq d \\
0 & x>d
\end{array},\right.
$$

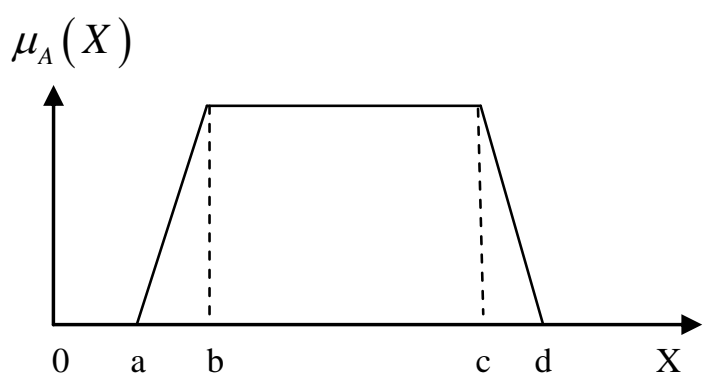

Fig. 2.1: Trapezoidal Fuzzy number

\subsection{Properties of Trapezoidal Fuzzy Number}

- Trapezoidal fuzzy number $\tilde{A}=(\mathrm{a}, \mathrm{b}, \mathrm{c}, \mathrm{d})$ is said to be non negative trapezoidal fuzzy number if and only if $a-c \geq 0$.

- A trapezoidal fuzzy number $\tilde{A}=(\mathrm{a}, \mathrm{b}, \mathrm{c}, \mathrm{d})$ is said to be zero trapezoidal fuzzy number if and only if $a=0$, $\mathrm{b}=0, \mathrm{c}=0, \mathrm{~d}=0$.

- Two trapezoidal fuzzy numbers $\tilde{A}=\left(\mathrm{a}_{1}, \mathrm{~b}_{1}, \mathrm{c}_{1}, \mathrm{~d}_{1}\right)$ and $\tilde{A}_{2}=\left(\mathrm{a}_{2}, \mathrm{~b}_{2}, \mathrm{c}_{2}, \mathrm{~d}_{2}\right)$ are said be equal i.e. $\tilde{A}_{1}=\tilde{A}_{2}$, if and only if $\mathrm{a}_{1}=\mathrm{a}_{2}, \mathrm{~b}_{1}=\mathrm{b}_{2}, \mathrm{c}_{1}=\mathrm{c}_{2}, \mathrm{~d}_{1=} \mathrm{d}_{2}$.

\subsection{Fuzzy Set}

A Fuzzy set A is defined as the set of ordered pairs $\left(X, \mu_{A}(X)\right)$, where $\mathrm{X}$ is an element of the universe of discourse $\mathrm{U}$ and $\mu_{A}(X)$ is the membership function, that attributes to each $X \in U$ a real number $\in[0,1]$, describing the degree to which $X$ belongs to the set.

\section{Example:}

Let $X=\{a, b, c, d\}$, Define $\mu_{\mathrm{A}}: \mathrm{X} \rightarrow[0,1]$ as follows:

$$
\mu_{\mathrm{A}}(\mathrm{a})=0, \mu_{\mathrm{A}}(\mathrm{b})=0.4, \quad \mu_{\mathrm{A}}(\mathrm{c})=0.6, \mu_{\mathrm{A}}(\mathrm{d})=1
$$

Then the class,

$A=\{(a, 0),(b, 0.4),(c, 0.6),(d, 1)\}$ is a fuzzy set on $X$.

\subsection{Crisp Set}

A crisp set is a special case of a Fuzzy set, in which the membership function only takes two values, commonly defined as 0 and 1 . 


\subsection{Arithmetic Operation [7]}

Let $A_{1}$ and $A_{2}$ be two trapezoidal fuzzy numbers parameterized by the quadruple $\left(a_{1}, a_{2}, a_{3}, a_{4}\right)$ and $\left(b_{1}, b_{2}, b_{3}, b_{4}\right)$, respectively. The simplified fuzzy number arithmetic operations between the trapezoidal fuzzy numbers $A_{1}$ and $A_{2}$ are as follows,

Fuzzy numbers addition $\oplus$

$$
\begin{aligned}
\left(a_{1}, a_{2}, a_{3}, a_{4}\right) \oplus & \left(b_{1}, b_{2}, b_{3}, b_{4}\right) \\
& =\left(a_{1}+b_{1}, a_{2}+b_{2}, a_{3}+b_{3}, a_{4}+b_{4}\right)
\end{aligned}
$$

Fuzzy numbers subtraction

$$
\begin{array}{r}
\rho=\frac{\vec{E}}{J_{C}(T=\text { const. }) \cdot\left(P \cdot\left(\frac{\vec{E}}{E_{C}}\right)^{m}+(1-P)\right)} \\
\left(a_{1}, a_{2}, a_{3}, a_{4}\right) \square\left(b_{1}, b_{2}, b_{3}, b_{4}\right) \\
=\left(a_{1}-b_{4}, a_{2}-b_{3}, a_{3}-b_{3}, a_{4}-b_{1}\right)
\end{array}
$$

Multiplication used by Stephen Dinegar.D \&Palanivel.K [15]:

$$
\begin{aligned}
\tilde{a} . \tilde{b}= & {\left[\frac{a_{1}}{4}\left(b_{1}+b_{2}+b_{3}+b_{4}\right), \frac{a_{2}}{4}\left(b_{1}+b_{2}+b_{3}+b_{4}\right),\right.} \\
& \left.\frac{a_{3}}{4}\left(b_{1}+b_{2}+b_{3}+b_{4}\right), \frac{a_{4}}{4}\left(b_{1}+b_{2}+b_{3}+b_{4}\right)\right], \text { 㕍 } R(\tilde{a})>0 \\
\tilde{a} . \tilde{b}=[ & \frac{a_{4}}{4}\left(b_{1}+b_{2}+b_{3}+b_{4}\right), \frac{a_{3}}{4}\left(b_{1}+b_{2}+b_{3}+b_{4}\right), \\
& \left.\frac{a_{2}}{4}\left(b_{1}+b_{2}+b_{3}+b_{4}\right), \frac{a_{1}}{4}\left(b_{1}+b_{2}+b_{3}+b_{4}\right)\right], \text { if } R(\tilde{a})<0
\end{aligned}
$$

\section{Example:}

Let $A_{1}$ and $A_{2}$ be two trapezoidal fuzzy numbers, where

$$
\begin{aligned}
& A_{1}=(1,2,3,4) \text { and } A_{2}=(5,6,7,8) . \text { Then, } \\
& A_{1} \oplus A_{2}=(1,2,3,4) \oplus(5,6,7,8)=(6,8,10,12) \\
& A_{1} \square A_{2}=(1,2,3,4) \square(5,6,7,8)=(-7,-5,-3,-1)
\end{aligned}
$$

\subsection{Mathematical Formulation of Fuzzy Transportation Problem}

Minimize: $\mathrm{Z}=\sum \sum \tilde{c}_{i j} \tilde{x}_{i j} \quad$ Subject to,

$$
\sum_{j=1}^{n} \tilde{x}_{i j} \leq \tilde{a}_{i} \text { for } \mathrm{i}=1,2, \ldots \ldots, \mathrm{m}
$$

$$
\begin{aligned}
& \sum_{i=1}^{m} \tilde{x}_{i j} \geq \tilde{b}_{j} \text { for } \mathrm{j}=1,2, \ldots ., \mathrm{n} \\
& \tilde{x}_{i j} \geq 0 \quad \text { for } \mathrm{i}=1,2, \ldots, \mathrm{m} \text { and } \mathrm{j}=1,2, \ldots, \mathrm{n}
\end{aligned}
$$

Where $\tilde{a}_{i}=\left(a_{1}, a_{2}, a_{3}, a_{4}\right), \tilde{b}_{j}=\left(b_{1}, b_{2}, b_{3}, b_{4}\right)$ and $\tilde{c}_{i j}=\left(c_{i j}, c_{i j}, c_{i j}, c_{i j}\right)$ representing the uncertain supply and demand for the transportation problem.

\subsection{Ranking Function [17]}

Many different approaches for the ranking of fuzzy numbers have been proposed in the literature. In this Fuzzy Russell's method we use Yager's[17] ranking method. A ranking function $\mathrm{R}: \mathrm{F}(\mathrm{R}) \rightarrow \mathrm{R}$ which maps each fuzzy number into the real line, $F(R)$ represents the set of all trapezoidal fuzzy number. If $\mathrm{R}$ be any ranking function,

$$
R(\tilde{a})=\frac{a_{1}+a_{2}+a_{3}+a_{4}}{4}
$$

For any two trapezoidal Fuzzy number $\tilde{A}=\left(a_{1}, a_{2}, a_{3}, a_{4}\right)$ and $\tilde{B}=\left(b_{1}, b_{2}, b_{3}, b_{4}\right)$ then we have,

$$
\begin{array}{lll}
\text { (i) } \tilde{A} \leq \widetilde{B} & \Leftrightarrow & R(\tilde{A}) \leq R(\widetilde{B}) \\
\text { (ii) } \tilde{A} \geq \widetilde{B} & \Leftrightarrow & R(\tilde{A}) \geq R(\widetilde{B}) \\
\text { (iii) } \tilde{A}=\widetilde{B} & \Leftrightarrow & R(\tilde{A})=R(\widetilde{B})
\end{array}
$$

\section{The Computational Procedure for Fuzzy Russell's Method}

In this section we proposes modified method called as Fuzzy Russell's method is used for finding initial basic feasible solution for Fuzzy transportation problem. The solution procedure as follows,

\subsection{Algorithm for Fuzzy Russell's Method}

Step 1: Calculate the quantities $\bar{u}_{i}, \bar{v}_{j}$ and $\square_{i j}$ using

$$
\begin{aligned}
& \overline{\boldsymbol{u}}_{\boldsymbol{i}}=\max _{1 \leq j \leq n}\left\{\boldsymbol{c}_{i j}\right\} \text { For } \mathrm{i}=1,2, \ldots \ldots \mathrm{m} \\
& \overline{\boldsymbol{v}}_{j}=\max _{1 \leq i \leq m}\left\{\boldsymbol{c}_{i j}\right\} \text { For } \mathrm{j}=1,2, \ldots \ldots \ldots \mathrm{n} \\
& \text { and } \square_{i j}=c_{i j}-\overline{\boldsymbol{u}}_{i}-\overline{\boldsymbol{v}}_{j} \text { For all } \mathrm{i}, \mathrm{j} .
\end{aligned}
$$

Step 2: Select the variables $x_{i j}\left(x_{\mathrm{ij}}{ }^{(1)}, x_{\mathrm{ij}}{ }^{(2)}, x_{\mathrm{ij}}{ }^{(3)}, x_{\mathrm{ij}}{ }^{(4)}\right)$ having the most negative value of $\square_{i j}$.If there are ties in the value of $\square_{i j}$,Select $x_{i j}\left(x_{\mathrm{ij}}{ }^{(1)}, x_{\mathrm{ij}}{ }^{(2)}, x_{\mathrm{ij}}{ }^{(3)}, x_{\mathrm{ij}}{ }^{(4)}\right)$ 
with the smallest unit $\operatorname{cost} \boldsymbol{c}_{\boldsymbol{i j}}\left(c_{\mathrm{ij}}^{(1)},{c_{\mathrm{ij}}}^{(2)}, c_{\mathrm{ij}}^{(3)}\right.$, $\left.c_{\mathrm{ij}}{ }^{(4)}\right)$.If there are ties again in the value of $c_{i j}\left(c_{\mathrm{ij}}{ }^{(1)}, c_{\mathrm{ij}}{ }^{(2)}, c_{\mathrm{ij}}{ }^{(3)}, c_{\mathrm{ij}}{ }^{(4)}\right)$, select $x_{i j}\left(x_{\mathrm{ij}}{ }^{(1)}, x_{\mathrm{ij}}{ }^{(2)}, x_{\mathrm{ij}}{ }^{(3)}\right.$, $\left.x_{\mathrm{ij}}{ }^{(4)}\right)$ with the largest amount of remaining source supply or destination demand.

Step3: Set the activity level of $x_{i j}\left(x_{\mathrm{ij}}^{(1)}, x_{\mathrm{ij}}^{(2)}, x_{\mathrm{ij}}{ }^{(3)}\right.$, $x_{\mathrm{ij}}{ }^{(4)}$ ) equal to the smaller value between the source supply $\bar{a}_{i}$ and the destination demand $\bar{b}_{j}$.

Step4: Subtract $x_{i j}\left(x_{\mathrm{ij}}^{(1)}, x_{\mathrm{ij}}^{(2)}, x_{\mathrm{ij}}^{(3)}, x_{\mathrm{ij}}^{(4)}\right)$ from $\bar{a}_{i}$ and $\bar{b}_{j}$ found in step3. Eliminate from the transportation table the row or column that results in a zero supply or destination demand after this subtraction. Stop if all $a_{i}(\mathrm{i}=1,2, \ldots, \mathrm{m})$ and $b_{j}(\mathrm{j}=1,2, \ldots, \mathrm{n})$ are zero, otherwise go to step1.

\section{Numerical Example}

Consider the Fuzzy transportation problem.

Here cost value, supplies and demands are trapezoidal fuzzy number. Here $\boldsymbol{c}_{\boldsymbol{i}}$ and $b_{j}$ are Fuzzy Supply and Fuzzy Demand. Fuzzy Russell's method is used to finding the initial basic feasible solution.

\begin{tabular}{cccccc}
\hline & $D_{1}$ & $D_{2}$ & $D_{3}$ & $D_{4}$ & $\boldsymbol{a}_{\boldsymbol{i}}$ \\
\hline$S_{1}$ & $(1,2,3,4)$ & $(1,3,4,6)$ & $(9,11,12,14)$ & $(5,7,8,11)$ & $(1,6,7,12)$ \\
$S_{2}$ & $(0,1,2,4)$ & $(-1,0,1,2)$ & $(5,6,7,8)$ & $(0,1,2,3)$ & $(0,1,2,3)$ \\
$S_{3}$ & $(3,5,6,8)$ & $(5,8,9,12)$ & $(12,15,16,19)$ & $(7,9,10,12)$ & $(5,10,12,15)$ \\
$b_{j}$ & $(5,7,8,10)$ & $(-1,5,6,10)$ & $(1,3,4,6)$ & $(1,2,3,4)$ & $(6,17,21,30)$ \\
\hline
\end{tabular}

Therefore $\sum_{i=1}^{m} a_{i}=\sum_{j=1}^{n} b_{j}$, the problem is balanced fuzzy transportation problem. There exists a fuzzy initial basic feasible solution.
Now we applying the Fuzzy Russell's method for fuzzy transportation problem we have,

\begin{tabular}{|c|c|c|c|c|c|}
\hline & $\mathbf{D}_{1}$ & $\mathbf{D}_{2}$ & $\mathbf{D}_{3}$ & $\mathbf{D}_{4}$ & $a_{i}$ \\
\hline$S_{1}$ & $(1,2,3,4)$ & $\begin{array}{l}(-5,2,4,11) \\
(1,3,4,6)\end{array}$ & $\begin{array}{l}(\mathbf{1}, \mathbf{3}, \mathbf{4 , 6}) \\
(9,11,12,14)\end{array}$ & $(5,7,8,11)$ & $(1,6,7,12)$ \\
\hline $\mathbf{S}_{2}$ & $(0,1,2,4)$ & $\begin{array}{l}(\mathbf{0}, \mathbf{1}, \mathbf{2}, \mathbf{3}) \\
(-1,0,1,2)\end{array}$ & $(5,6,7,8)$ & $(0,1,2,3)$ & $(0,1,2,3)$ \\
\hline$S_{3}$ & $\begin{array}{l}(\mathbf{5 , 7}, \mathbf{8 , 1 0}) \\
(3,5,6,8)\end{array}$ & $\begin{array}{l}(-15,-1,3,15) \\
(5,8,9,12)\end{array}$ & $(12,15,16,19)$ & $\begin{array}{l}(\mathbf{1}, \mathbf{2}, \mathbf{3}, \mathbf{4}) \\
(7,9,10,12)\end{array}$ & $(5,10,12,15)$ \\
\hline $\mathbf{b}_{\mathbf{j}}$ & $(5,7,8,10)$ & $(-1,5,6,10)$ & $(1,3,4,6)$ & $(1,2,3,4)$ & $(6,17,21,30)$ \\
\hline
\end{tabular}

Therefore the initial basic feasible solution is,

$$
\text { Minimum } Z\left(Z^{(1)}, Z^{(2)}, Z^{(3)}, Z^{(4)}\right)
$$

$=[(-5,2,4,11)(1,3,4,6)]+[(0,1,2,3)(-1,0,1,2)]+$ $[(5,7,8,10)(3,5,6,8)]+[(5,7,8,10)(3,5,6,8)]+[(-15,-1$, $3,15)(5,8,9,12)]+[(1,2,3,4)(7,9,10,12)]$.

$$
\begin{aligned}
& \operatorname{Minimum} Z\left(Z^{(1)}, Z^{(2)}, Z^{(3)}, Z^{(4)}\right) \\
& =(158.25,90.5,158.25,328.5)
\end{aligned}
$$

The crisp value of the Fuzzy Transportation problem is 183.875 .

\section{Conclusion}

We proposed Fuzzy Russell's method to find the initial basic feasible solution using Yager's ranking method with trapezoidal fuzzy numbers. This method can be used for all kinds of fuzzy numbers. This method is very easy to apply and can be utilized for the fuzzy transportation problem. This technique can also be tried in solving other types of problem like, project schedules, assignment problem and network flow problem. 


\section{Acknowledgment}

The authors would like to heartily thank the Editor in Chief and anonymous reviewers for their careful reading of this paper and for their helpful comments.

\section{References}

[1] Amarpreet kaur,Amit Kumar. 'A new method for solving fuzzy transportation problem using ranking function', Applied Mathematical Modeling, (2011), 35, pp: 5652-5661.

[2] Chanas, S and Kuchta, D. 'A concept of the optimal solution of the transportation problem with fuzzy cost coefficients', Fuzzy sets and Systems, (1996) ,82,pp: 299-305.

[3] Chanas.S, Kolodziejczyk.W and Machaj, A. 'A fuzzy approach to the transportation problem', Fuzzy Sets and Systems, (1984), 13, pp: 211-221.

[4] Campos, L. and Gonzalez Munoz, A. 'A subjective approach for ranking fuzzy number', Fuzzy Sets and Systems, (1989), 29, pp: 145-153.

[5] Campos, L. and Verdegay, J. L. 'Linear programming problem and ranking of fuzzy numbers', Fuzzy Sets and Systems, (1989),32,pp $1-11$.

[6] F.L.Hitchcock. 'The distribution of a product from several source to numerous localities', J.Math.phys, (1941), 20,pp:224-230.

[7] Ismail Mohideen, S. and Senthil Kumar, P. 'A Comparative Study on Transportation Problem in Fuzzy Environment', International Journal of Mathematics Research, ISSN 0976-5840, (2010), Vol.2, Number 1, pp: 151-158.

[8] Jain, R. 'Decision-making in the presence of fuzzy variables', IEEE Transactions on Systems, Man and Cybernetics, (1976), 6, pp: 698-703.

[9] Kim, K. and Park, K. S. 'Ranking fuzzy number with index of optimism', Fuzzy Sets and Systems, (1990), 35, pp: 143-150.

[10] Liou, T. S. and Wang, M. J. 'Ranking fuzzy numbers with integral value', Fuzzy Sets and Systems, (1992), 50, pp: 247-255.

[11] Nagoor Gani, A. and Abdul Razak, K. 'Two Stage Fuzzy Transportation Problem', Journal of Physical Sciences, (2006), Vol.10,pp:63-69.

[12] Narayanamoorthy, S., Anukokila P. 'Robust fuzzy transportation problems based on extension principle under uncertain Demands', IJMA, (2012), Vol.5.No.1. pp: 01-19.

[13] Pandian, P and Natarajan, G. 'A new algorithm for finding a fuzzy optimal solution for fuzzy transportation problem', Applied Mathematical Sciences, (2010), 4, pp: 79-90.

[14] Shiang-Tai Liu, and Chiang Kao. 'Solving fuzzy transportation problem based on extension principle', Journal of Physical Science, (2006), 10, pp: 63-69.

[15] Stephen Dinagar, D and Palanivel, K., 'The transportation problem in Fuzzy Environment'. International Journal of Algorithms, Computing and Mathematics, August 2009, Vol 2, Number 3.

[16] Tze-San lee. 'A complete Russell's method for the Transportation Problem', (1986), Vol.28, No.4.

[17] Yager, R.R. 'A procedure for ordering fuzzy subsets of the unit interval', Information Sciences, (1981), 24, pp: 143-161.

[18] Zadeh, L. A. Fuzzy sets, Information and Control, (1965), 8, pp: 338-353.

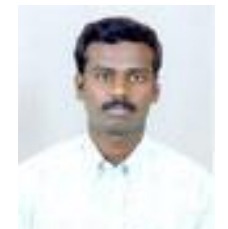

S. Narayanamoorthy was born in Tamilnadu, India in 1979. He received the B.Sc., M.Sc., M.Phil., \& Ph.D., degrees from Loyola College (Autonomous), Chennai, India, in $1999,2001,2002$ and 2008 respectively. In 2009, he joined the Bharathiar University, Coimbatore, Tamilnadu, India, as an Assistant Professor, Department of Applied Mathematics. His activities currently focus on Applications of Fuzzy Mathematics (Social Problem). His research interest includes Fuzzy Optimization, Fuzzy Differential Equations and Fuzzy Graphs.

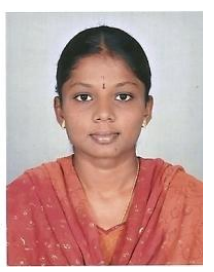

S. Saranya was born in Tamilnadu, India in 1989. She received the B.Sc., degree from PKR Arts and Science College, Gobichettipalayam, Erode, in 2009 and M.Sc., M.Phil., degrees from Bharathiar University, Coimbatore, Tamilnadu, India. Now she is doing her research in Fuzzy Optimization.

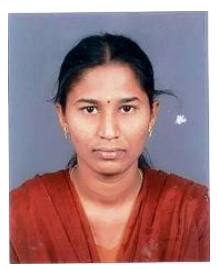

S. Maheswari was born in Tamilnadu, India in 1989.She received the B.Sc., degree from Selvam Arts and Science College, Namakkal, in 2009 , and M.Sc., M.Phil degrees from Bharathiar University, Coimbatore, Tamilnadu, India. Now she is doing her research in Fuzzy Optimization. 